\title{
Hartree-Bose mean-field approximation for the interacting boson model (IBM-3)
}

\author{
J. E. García-Ramos, ${ }^{1}$ J. M. Arias, ${ }^{1}$ J. Dukelsky, ${ }^{2}$ E. Moya de Guerra, ${ }^{2}$ and P. Van Isacker $^{3}$ \\ ${ }^{1}$ Departamento de Física Atómica, Molecular y Nuclear, Universidad de Sevilla, Apartado 1065, 41080 Sevilla, Spain \\ ${ }^{2}$ Instituto de Estructura de la Materia, Serrano 123, 28006 Madrid, Spain \\ ${ }^{3}$ Grand Accélérateur National d'Ions Lourds, Boîte Postal 5027, F-14076 Caen Cedex 5, France
}

(Received 9 May 1997)

\begin{abstract}
A Hartree-Bose mean-field approximation for the IBM-3 is presented. A Hartree-Bose transformation from spherical to deformed bosons with charge-dependent parameters is proposed which allows bosonic pair correlations and includes higher angular momentum bosons. The formalism contains previously proposed IBM-2 and IBM-3 intrinsic states as particular limits. [S0556-2813(98)50202-6]
\end{abstract}

PACS number(s): 21.60.Fw, 21.60.-n, 21.60.Ev

With the advent of radioactive nuclear beam (RNB) facilities, unexplored regions of the nuclear chart will become available for spectroscopic studies. New aspects of nuclear dynamics and novel types of collectivity and nuclear topologies are expected. Nuclei with roughly equal numbers of protons and neutrons $(Z \sim N)$ and with masses in between ${ }^{40} \mathrm{Ca}$ and ${ }^{100} \mathrm{Sn}$ are of particular interest because the buildup of nuclear collectivity in this mass region occurs in the presence of pairing correlations between alike nucleons (protonproton and neutron-neutron) as well as between neutrons and protons [1]. This offers the possibility of experimentally accessing nuclei that exhibit a superconducting phase arising from proton-neutron Cooper pairs [2]. Although recent breakthroughs $[3,4]$ have made shell-model calculations possible for this mass region, they are still of a daunting complexity and alternative approximation schemes are required that yield a better intuitive (e.g., geometric) insight.

One of the possible alternatives is the interacting boson model (IBM) [5]. It has been shown [6] that nuclei with protons and neutrons filling the same valence shell require an extended boson model, IBM-3. In IBM-3 three types of bosons are included: proton-proton $(\pi)$, neutron-neutron $(\nu)$, and proton-neutron $(\delta)$. The $\pi, \nu$, and $\delta$ bosons are the three members of a $T=1$ triplet, and their inclusion is necessary to obtain an isospin-invariant formulation of the IBM. Over the last decade the validity of the IBM-3 has been tested and its relationship with the shell model worked out $[7-11]$.

The mean-field formalism has been an important tool to acquire a geometric understanding of the IBM ground state and of the vibrations around the deformed equilibrium shape [12-14]. Moreover, a treatment based on mean-field techniques generally leads to a considerable reduction in the complexity of the calculation, allowing the introduction of additional degrees of freedom if needed. Studies in the intrinsic framework are thus useful to assess the importance of higher angular momentum bosons with, e.g., $\ell$ $=3^{-}, 4^{+}, \ldots$ or to investigate the role of extra degrees of freedom not included in IBM such as two-quasiparticle excitations, etc.

An intrinsic-state formalism for the IBM-3 was recently presented by Ginocchio and Leviatan (GL) [15]. In that work charge-independent deformation parameters are imposed in the Hartree-Bose transformation from spherical to axially de- formed bosons and the trial wave function is taken to have good isospin. Closer inspection reveals that this trial wave function has the additional isospin SU(3) symmetry which in IBM-3 is equivalent to orbital $\mathrm{U}(6)$ symmetry. [Isospin $\mathrm{SU}(3)$ symmetry is to IBM-3 what $F$-spin symmetry [16] is to IBM-2.] Moreover, it is well known that isospin symmetry itself is increasingly broken in $Z \sim N$ nuclei as the nuclear mass increases $[17,18]$. There is also tentative evidence for rigid triaxial shapes in the region of interest and its proper description would require the inclusion of three body forces or higher angular momentum bosons. We therefore present in this paper a generalization of the treatment of GL in which none of the above symmetries [isospin $\mathrm{SU}(3)$ and $\mathrm{SU}(2)$ ] is imposed on the trial wave function and which includes bosons of angular momenta higher than $\ell=2$. For practical applications we restrict ourselves here to $\ell=0,2$.

We start with the usual spherical boson creation and annihilation operators $\gamma_{\ell m \tau}^{\dagger}, \gamma_{\ell m \tau}$, where $\ell$ is the angular momentum, $m$ is its third component, and $\tau$ is the isospin projection. Each boson carries isospin $T=1$. We also define $\tilde{\gamma}_{\ell m \tau}=(-1)^{\ell-m} \gamma_{\ell-m \tau}$. In terms of these boson operators, a system of $N$ bosons interacting through a general numberconserving two-body Hamiltonian can be written in multipolar form as

$$
H=\sum_{\ell \tau} \varepsilon_{\ell \tau} \gamma_{\ell \tau}^{\dagger} \cdot \tilde{\gamma}_{\ell \tau}+\sum_{L} \sum_{\tau_{1} \tau_{2} \tau_{3} \tau_{4}} \kappa_{\tau_{1} \tau_{2} \tau_{3} \tau_{4}}^{L} \hat{T}_{\tau_{1} \tau_{2}}^{L} \cdot \hat{T}_{\tau_{3} \tau_{4}}^{L},
$$

where the center dot denotes scalar product in orbital space. In isospin space the only restriction is $\tau_{1}+\tau_{2}=\tau_{3}+\tau_{4}$ (i.e., a charge-conserving Hamiltonian is assumed) and $\hat{T}_{\tau_{1} \tau_{2}}^{L}$ are multipole operators with total angular momentum $L$,

$$
\hat{T}_{M, \tau_{1} \tau_{2}}^{L}=\sum_{\ell_{1} \ell_{2}} \chi_{\ell_{1} \ell_{2}, \tau_{1} \tau_{2}}^{L}\left(\gamma_{\ell_{1} \tau_{1}}^{\dagger} \times \tilde{\gamma}_{\ell_{2} \tau_{2}}\right)_{M}^{L},
$$

where the coupling is only done in angular momentum. The Hamiltonian (1) can be used for IBM-3, for IBM-2, or even for a general isospin nonconserving Hamiltonian with three kinds of bosons.

Deformed bosons are defined in terms of spherical ones by means of a unitary Hartree-Bose transformation 


$$
\Gamma_{p \tau}^{\dagger}=\sum_{\ell m} \eta_{\ell m}^{p \tau} \gamma_{\ell m \tau}^{\dagger}, \quad \gamma_{\ell m \tau}^{\dagger}=\sum_{p} \eta_{\ell m}^{* p \tau} \Gamma_{p \tau}^{\dagger},
$$

and their Hermitian conjugates. The deformation parameters $\eta_{/ m}^{p \tau}$ in these equations verify the orthonormalization conditions

$$
\sum_{\ell m} \eta_{\ell m}^{* p^{\prime} \tau} \eta_{\ell m}^{p \tau}=\delta_{p p^{\prime}}, \quad \sum_{p} \eta_{\ell m}^{* p \tau} \eta_{\ell^{\prime} m^{\prime}}^{p \tau}=\delta_{\ell \ell^{\prime}} \delta_{m m^{\prime}}
$$

Note the explicit dependence on the isospin component $\tau$ of the transformation $\eta$, allowing different structures for the different condensed bosons $\pi, \nu$, and $\delta$. The index $p$ labels different possible deformed bosons. We choose $p=0$ for the fundamental deformed bosons and $p=1,2, \ldots$ for the different excited bosons. For instance, in an SU(3) scheme different values of $p=0,1,2,3$ label the ground, $\beta, \gamma$, and scissors bands, respectively. Since in this work we only treat the ground-state condensed boson, the Hartree superscript $p$ is always zero here and it will be omitted in the following. The formalism for the excited states will be presented elsewhere.

Following Ref. [15], the trial wave function for the ground state of an even-even system with a proton excess is of the form (the trial wave function for an even-even system with a neutron excess is obtained by interchanging the role of protons and neutrons)

$$
|\phi(\alpha)\rangle=\Lambda^{\dagger N_{n}}(\alpha) \Gamma_{1}^{\dagger N_{p}-N_{n}|0\rangle,}
$$

where the operator $\Lambda^{\dagger}$ creates a correlated bosonic pair in isospin space

$$
\Lambda^{\dagger}(\alpha)=\Gamma_{1}^{\dagger} \Gamma_{-1}^{\dagger}+\alpha \Gamma_{0}^{\dagger} \Gamma_{0}^{\dagger} .
$$

In Eq. (5) $N_{p}\left(N_{n}\right)$ is the number of proton (neutron) pairs in the valence space. The trial wave function (5) contains the isospin-conserving formalism of GL and the IBM-2 as natural limits. Two different values of $\alpha$ are connected with these limits. For $\alpha=-\frac{1}{2}, \Lambda^{\dagger}(\alpha)$ corresponds, in the particular case of $\tau$-independent deformation parameters, to an isoscalar bosonic pair. Its total isospin is $T=N_{p}-N_{n}$ and the results of GL are reproduced. Any other value of $\alpha$ breaks isospin symmetry. In particular, $\alpha=0$ eliminates the mixing of $\delta$ bosons in the ground state and yields an IBM-2 intrinsic state. It should be emphasized that when the deformation parameters $\eta_{\ell m}^{p \tau}$ in Eq. (3) depend on the isospin component $\tau$, the set of operators $\Gamma_{p \tau}^{\dagger}$ with $\tau=-1,0,1$ do not form an isospin triplet, and consequently the $\Lambda^{\dagger}$ in Eq. (6) may contain mixtures of $T=0,1,2$ isospin components.

At this point we would like to remark that the trial wave function (5) is not the most general U(18) intrinsic state, involving a combination of all $\tau=-1,0,1$ condense bosons. The U(18) intrinsic state is written as

$$
|\phi\rangle_{\mathrm{U}(18)}=\left(\Gamma_{c}^{\dagger}\right)^{N_{p}+N_{n}}|0\rangle
$$

where

$$
\Gamma_{c}^{\dagger}=\sum_{\ell m \tau} \xi_{\ell m \tau} \gamma_{\ell m \tau}^{\dagger} .
$$

In this state orbital angular momentum, isospin, and charge are broken. The state given in Eq. (5) improves over this state by including charge conserving pair correlations. Thus, it is expected to lead to a deeper energy minimum. Numerical results illustrating this point will be presented later on.

The variational parameters of the trial wave function are the matrix elements $\eta_{\ell m}^{\tau}$ of the Hartree-Bose transformation, associated with the orbital and isospin degrees of freedom, and the parameter $\alpha$, which determines the amount of mixing of $\delta$ bosons in the ground state.

The ground-state energy is obtained by taking the expectation value of the Hamiltonian (1) in the state (5):

$$
\begin{aligned}
E(\eta, \alpha)= & \sum_{\tau} \epsilon_{\tau} f_{1}(\alpha, \tau) \\
& +\sum_{\tau_{1} \tau_{2} \tau_{3} \tau_{4}} V_{\tau_{1}, \tau_{2}, \tau_{3}, \tau_{4}}^{c} f_{2}\left(\alpha, \tau_{1} \tau_{2} \tau_{3} \tau_{4}\right),
\end{aligned}
$$

where

$$
\begin{aligned}
& \epsilon_{\tau}=\sum_{\ell m} \widetilde{\varepsilon}_{\ell \tau} \eta_{\ell m}^{* \tau} \eta_{\ell m}^{\tau} \\
& V_{\tau_{1}, \tau_{2}, \tau_{3}, \tau_{4}}^{c}=\sum_{\ell_{1} m_{1} \ell_{2} m_{2} \ell_{3} m_{3} \ell_{4} m_{4}}
\end{aligned}
$$

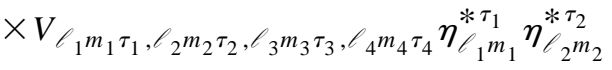

$$
\begin{aligned}
& \times \eta_{\ell_{3} m_{3}}^{\tau_{3}} \eta_{\ell}^{\tau_{4} m_{4}} \\
& f_{1}(\alpha, \tau)=\frac{\left\langle\phi(\alpha)\left|\Gamma_{\tau}^{\dagger} \Gamma_{\tau}\right| \phi(\alpha)\right\rangle}{\langle\phi(\alpha) \mid \phi(\alpha)\rangle},
\end{aligned}
$$

and

$$
f_{2}\left(\alpha, \tau_{1} \tau_{2} \tau_{3} \tau_{4}\right)=\frac{\left\langle\phi(\alpha)\left|\Gamma_{\tau_{1}}^{\dagger} \Gamma_{\tau_{2}}^{\dagger} \Gamma_{\tau_{3}} \Gamma_{\tau_{4}}\right| \phi(\alpha)\right\rangle}{\langle\phi(\alpha) \mid \phi(\alpha)\rangle} .
$$

The coefficients $\widetilde{\varepsilon}_{\ell \tau}$ include the single particle energies $\varepsilon_{\ell} \tau$ in Eq. (1) plus contributions from the two body term in the

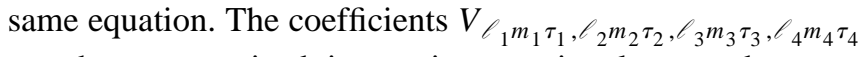
are the symmetrized interaction matrix elements between normalized two-boson states following Ref. [14],

$$
\begin{aligned}
& V_{\ell_{1} m_{1} \tau_{1}, \ell_{2} m_{2} \tau_{2}, \ell_{3} m_{3} \tau_{3}, \ell_{4} m_{4} \tau_{4}}=\frac{1}{4}\left\langle\ell_{1} m_{1} \tau_{1}, \ell_{2} m_{2} \tau_{2}|H| \ell_{3} m_{3} \tau_{3}, \ell_{4} m_{4} \tau_{4}\right\rangle \\
& \quad \times \sqrt{1+\delta_{\ell_{1} \ell_{2}} \delta_{m_{1} m_{2}} \delta_{\tau_{1} \tau_{2}}} \sqrt{1+\delta_{\ell_{3} \ell_{4}} \delta_{m_{3} m_{4}} \delta_{\tau_{3} \tau_{4}}} .
\end{aligned}
$$

The dependence of the energy on the variational parameters $\eta$ 's is contained in the one-body $\epsilon$ (10) and the twobody $V^{c}$ (11) terms, while the dependence on $\alpha$ comes through the isospin matrix elements $f_{1}(12)$ and $f_{2}(13)$. The latter matrix elements are straightforward to calculate by a binomial expansion of the ground-state trial wave function (5).

The Hartree-Bose equations for the orbital variational parameters $\eta$ are obtained by minimizing the energy (9) constrained by the norm of the transformation. Assuming a charge-conserving Hamiltonian (1), the following HartreeBose equations result: 


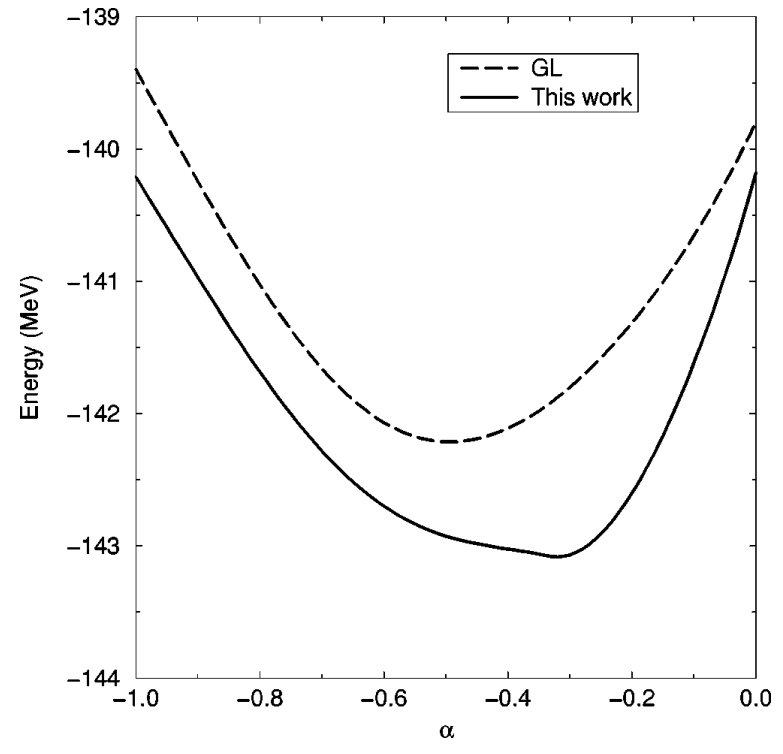

FIG. 1. Calculated ground-state intrinsic energy as a function of $\alpha$ for a system with 5 proton and 3 neutron pairs interacting through the Ginocchio Hamiltonian (17) with $\kappa=1 \mathrm{MeV}$.

$$
\sum_{\ell_{2} m_{2}} h_{\ell_{1} m_{1}, \ell_{2} m_{2}}^{\tau} \eta_{\ell_{2} m_{2}}^{\tau}=E_{\tau} \eta_{\ell_{1} m_{1}}^{\tau}
$$

where the Hartree-Bose matrix $h^{\tau}$ is

$$
\begin{aligned}
h_{\ell_{1} m_{1}, \ell_{2} m_{2}}^{\tau}= & \tilde{\varepsilon}_{\ell_{1} \tau} f_{1}(\alpha, \tau) \delta_{\ell_{1} \ell_{2}} \delta_{m_{1} m_{2}} \\
& +2 \sum_{{ }_{3} m_{3} \ell_{4} m_{4} \tau_{2} \tau_{3} \tau_{4}} V_{\ell_{1} m_{1} \tau, \ell_{3} m_{3} \tau_{3}, \ell_{4} m_{4} \tau_{4}, \ell_{2} m_{2} \tau_{2}} \\
& \times \frac{\eta_{\ell_{3} m_{3}}^{* \tau_{3}} \eta_{\ell} \tau_{4} m_{4} \eta_{\ell_{2} m_{2}}^{\tau_{2}}}{\eta_{\ell}^{\tau}{ }_{2} m_{2}} f_{2}\left(\alpha, \tau \tau_{3} \tau_{4} \tau_{2}\right) .
\end{aligned}
$$

The term $\eta_{\ell_{2} m_{2}}^{\tau}$ in the denominator is a consequence of a mathematical trick for obtaining a set of three coupled Hartree-Bose equations (15). These depend on the isospin matrices $f_{1}$ and $f_{2}$. For each value of $\alpha$ the matrices $f_{1}$ and $f_{2}$ are calculated and the Hartree-Bose Eqs. (15)-(16) are solved self-consistently. The procedure is iterated until one finds the absolute minimum of the energy (9). Once the problem is solved self-consistently, the diagonalization of (15) provides the deformation parameters $\eta_{\ell m}^{\tau}$ for the ground state.

To test the present formalism and to compare with the one by GL, we used a simple Hamiltonian recently proposed by Ginocchio [19],

$$
H=-\kappa \sum_{T=0,1,2} \hat{P}^{T}: \hat{P}^{T}
$$

where

$$
\hat{P}^{T}=\left[s^{\dagger} \widetilde{\widetilde{d}}+(-1)^{T} d^{\dagger} \widetilde{s^{2}}\right]^{L=2, T} .
$$

In these equations the colon denotes a scalar product in orbital and isospin spaces and $\widetilde{\widetilde{\gamma}}_{\ell m \tau}$ $=(-1)^{\ell-m+1-\tau} \gamma_{\ell-m-\tau}$. The Hamiltonian (17) is clearly isospin invariant and provides a first simple test to the present formalism.

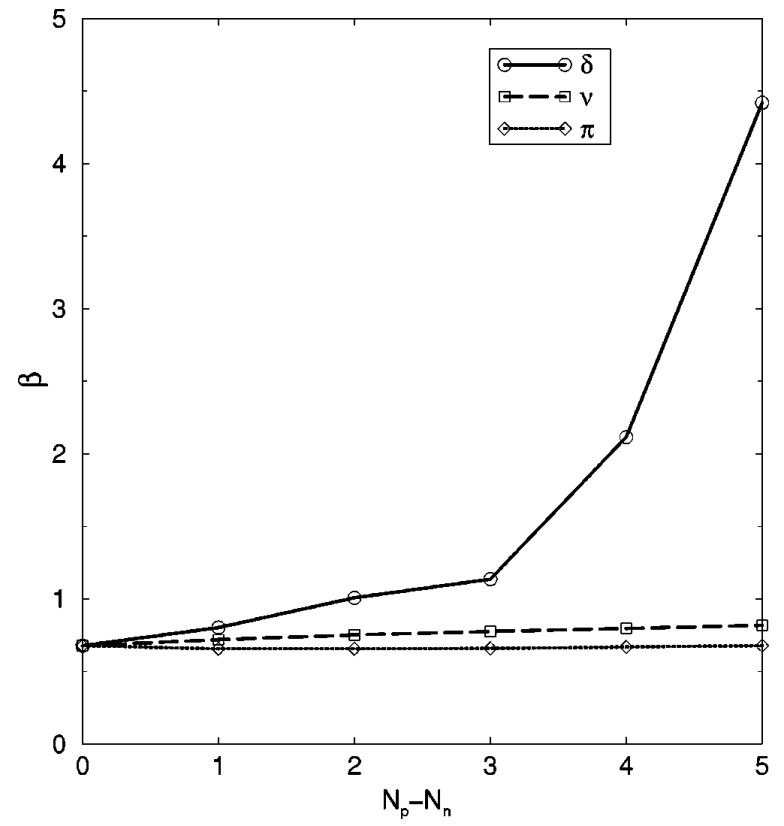

FIG. 2. Deformation parameters $\beta_{\tau}$ for a system with $N_{n}=4$ neutron pairs as a function of the difference $N_{p}-N_{n}$ between the numbers of proton and neutron pairs. The Ginocchio Hamiltonian (17) with $\kappa=1 \mathrm{MeV}$ is used.

Figure 1 shows, for a system with 5 proton pairs and 3 neutron pairs, the ground-state energy for the Hamiltonian (17) as a function of $\alpha$. The dashed line is calculated with $\tau$-independent deformation parameters; the GL minimum energy is reproduced for $\alpha=-\frac{1}{2}$. The full line is calculated with the present formalism. The latter calculation always gives a lower energy and, in particular, the minimum is not obtained for $\alpha=-\frac{1}{2}$, but for $\alpha \approx-0.32$. In addition, the corresponding deformation parameters are $\tau$ dependent. The energy gained by breaking isospin invariance in our trial wave

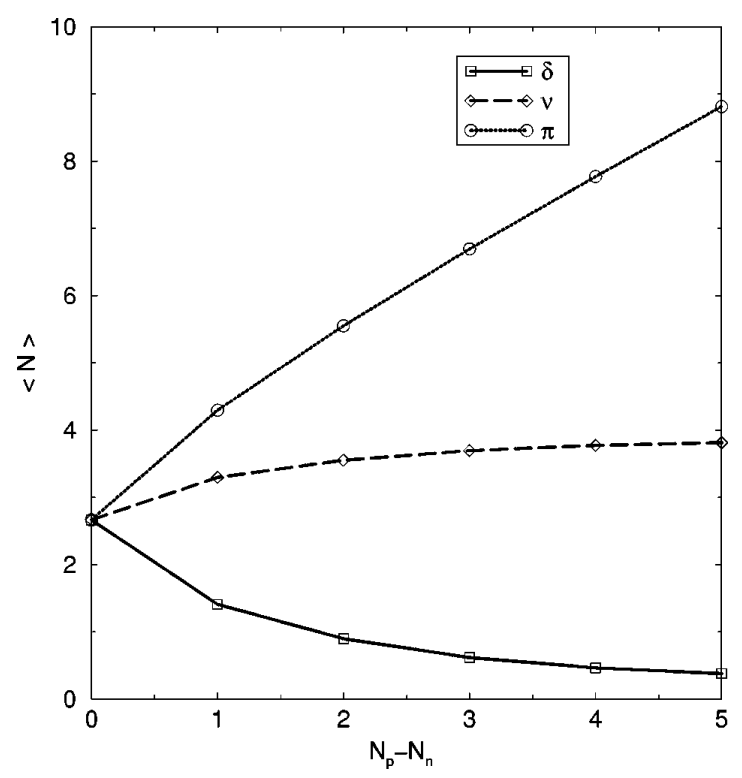

FIG. 3. Mean values of the boson numbers, $N_{\tau}$, for a system with $N_{n}=4$ neutron pairs as a function of the difference $N_{p}-N_{n}$ between the numbers of proton and neutron pairs. The Ginocchio Hamiltonian (17) with $\kappa=1 \mathrm{MeV}$ is used. 


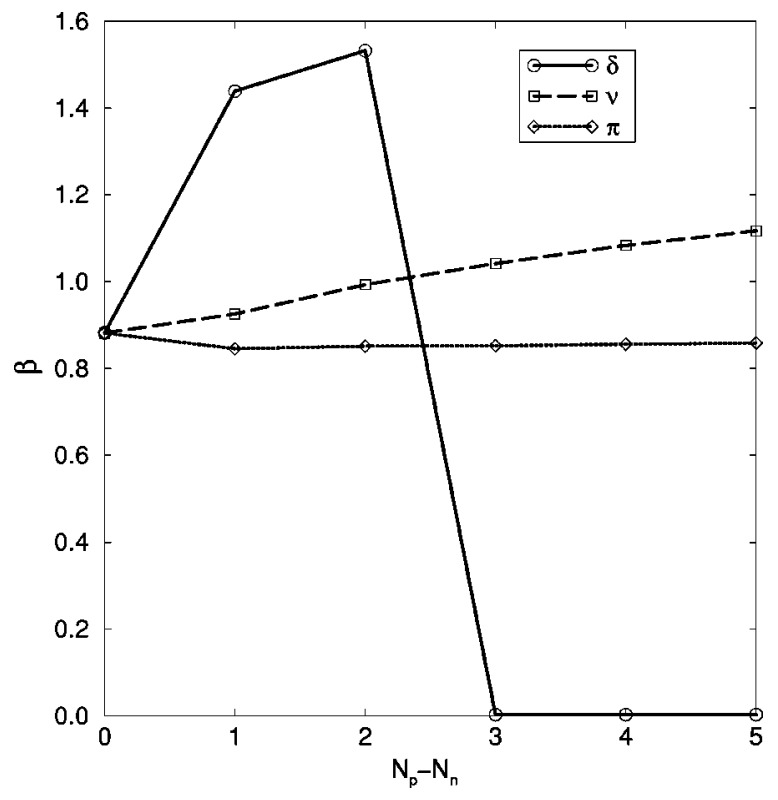

FIG. 4. Same as Fig. 2, but for the nonconserving isospin Hamiltonian (20) with the parameters given in the text.

function is relatively small. In this respect it may be advantageous to use the GL intrinsic state for isospin conserving Hamiltonians. Though, a better approximation would be obtained by performing variation after isospin projection over our trial wave functions.

We note that for a system with equal number of protons and neutrons, the present formalism recovers exactly the GL results; differences occur for $Z \neq N$. This can be seen in Fig. 2 where the deformation parameters $\beta_{\tau}$ are plotted versus the difference $N_{p}-N_{n}$ (starting with 4 proton pairs and 4 neutron pairs). The deformation parameters $\beta_{\tau}$ are obtained from $\beta_{\tau}=\sqrt{\left(1 /\left|\eta_{00}^{0 \tau}\right|^{2}\right)-1}$ [see Eq. (1) of Ref. [15]]. For $N_{p}=N_{n}$ the deformation parameters are independent of $\tau$, but not any longer as $N_{p}-N_{n}$ increases. The proton and neutron deformations remain very close; the $\delta$ deformation $\beta_{\delta}$, however, quickly becomes very large in comparison. This is because $N_{\delta}$ decreases as $N_{p}-N_{n}$ increases. This effect can be seen in Fig. 3 where the mean values of the boson numbers, $N_{\tau}$, are plotted. The same behavior has been obtained recently with large scale shell model calculations (see Ref. [20]). In all our calculations we found that the Ginocchio Hamiltonian (17) leads to a $\gamma$-independent energy surface.

It is worth noting that the present formalism allows one to reproduce the well-known case of triaxiality in IBM-2. To show this we use the IBM-2 Hamiltonian

$$
H=-\left(Q_{\pi}+Q_{\nu}^{\prime}\right) \cdot\left(Q_{\pi}+Q_{\nu}^{\prime}\right)
$$

where the center dot denotes scalar product in angular momentum, $Q_{\pi}$ is the $\mathrm{SU}(3)$ generator, $Q=s^{\dagger} \widetilde{d}$ $+d^{\dagger} \widetilde{s}-(\sqrt{7} / 2)\left(d^{\dagger} \times \widetilde{d}\right)^{L=2}$, for proton bosons and $Q_{\nu}^{\prime}$ is the $\overline{\mathrm{SU}(3)}$ generator, $\quad Q^{\prime}=s^{\dagger} \widetilde{d}+d^{\dagger} \widetilde{s}+(\sqrt{7} / 2)\left(d^{\dagger} \times \widetilde{d}\right)^{L=2}$, for neutron bosons. The minimization procedure now gives $\alpha=0$, which corresponds to the IBM-2 limit. In addition, the minimum deformation parameters correspond to a prolate proton condensate, axially symmetric about the intrinsic $z$ axis, and to an oblate neu-

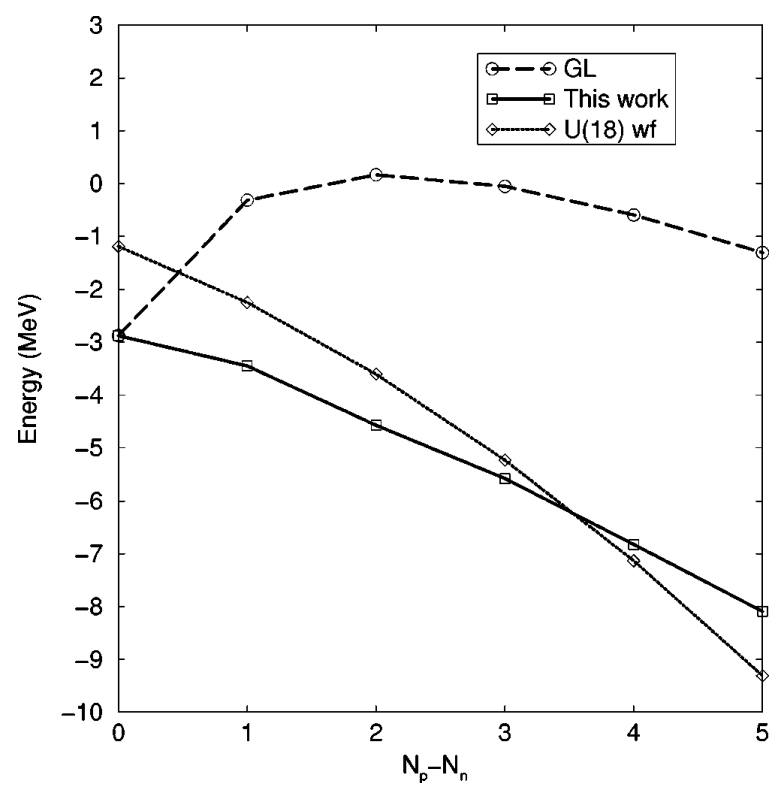

FIG. 5. Calculated ground-state intrinsic energy, as a function of the difference $N_{p}-N_{n}$ between the numbers of proton and neutron pairs, for the nonconserving isospin Hamiltonian (20) with the parameters given in the text.

troncondensate axially symmetric about the intrinsic $y$ axis, giving rise to an overall triaxial shape. It should be pointed out that in this case there is no triaxial minimum for aligned proton-neutron shapes with equal deformations. Here the overall shape is triaxial, but the underlying separate proton and neutron condensates correspond to different (prolateoblate) axial shapes.

Finally, we present a calculation in which isospin is explicitly broken by the Hamiltonian:

$$
H=\sum_{\ell \tau} \epsilon_{\ell \tau} \hat{n}_{\ell \tau}-\frac{1}{5} N\left[Q^{0}: Q^{0}+\frac{2}{3} Q^{1}: Q^{1}\right],
$$

where $Q^{T}=\left[s^{\dagger} \widetilde{\widetilde{d}}+d^{\dagger} \widetilde{\widetilde{s}}-(\sqrt{7} / 2)\left(d^{\dagger} \times \widetilde{\widetilde{d}}\right)\right]^{L=2, T}$ and $N[\ldots]$ stands for normal ordering product. In Figs. 4 and 5 we present the results of a calculation with $\epsilon_{s \pi}=\epsilon_{s \nu}=0, \epsilon_{d \pi}$ $=\epsilon_{d \nu}=1.5, \epsilon_{s \delta}=2.3\left|N_{p}-N_{n}\right|$, and $\epsilon_{d \delta}=1.5+2.3\left|N_{p}+N_{n}\right|$ (all $\epsilon$ 's in MeV). In Fig. 4 the deformation parameters, $\beta$ 's, are shown as a function of $N_{p}-N_{n}$ (the calculation starts with $N_{p}=N_{n}=4$ and then $N_{p}$ is increased). Figure 5 shows the corresponding ground-state energies for the intrinsic states of GL, and those defined in Eqs. (5) and (7). It is interesting to note that the GL intrinsic state and our pair correlated intrinsic state produce the same results for $N_{p}$ $-N_{n}=0$, being better than the $\mathrm{U}(18)$ intrinsic state. For $N_{p}$ $-N_{n}>0$ both isospin nonconserving intrinsic states give better results than GL. It is also interesting to see that our pair correlated ansatz is superior to the $\mathrm{U}(18)$ for moderate values of $N_{p}-N_{n}$. No triaxial deformation is found in these calculations.

In summary, we have extended the intrinsic-state formalism of Ginocchio and Leviatan [15] for IBM-3 in three different ways. First, the Hartree-Bose transformation is chosen to depend on the isospin component $\tau$. Second, variable isospin bosonic pair correlations are introduced through the parameter $\alpha$. Finally, higher-order bosons, other than the usual 
$s$ and $d$ bosons, are included in the Hartree-Bose transformation. This formalism contains the IBM-2 and GL intrinsic states as particular limits. Substantial differences in the deformation parameters are obtained when $N_{p} \neq N_{n}$. We have presented results for isospin conserving and nonconserving Hamiltonians with $s$ and $d$ bosons. Substantial differences in the deformation parameters $\beta_{\tau}$ are obtained for $N_{p}>N_{n}$. In most of the cases studied, our pair correlated intrinsic ground states are lower in energy than the GL ground states, al- though for the isospin conserving Hamiltonian the energy gain is small. We therefore conclude that the new intrinsic state is useful for treating isospin breaking Hamiltonians.

This work has been supported in part by the Spanish DGICYT under Contract Nos. PB95/0123 and PB95-0533, a DGICYT-IN2P3 agreement and by the European Commission under Contract CI1*-CT94-0072.
[1] D. D. Warner, in Perspectives for the Interacting Boson Model, edited by R.F. Casten et al. (World Scientific, Singapore, 1994), p. 373.

[2] W. Nazarewicz and S. Pittel, http://www.aps.org/ BAPSAPR97/vpr/laye8.html

[3] S. E. Koonin, D. J. Dean, and K. Langanke, Phys. Rep. (to be published).

[4] M. Honma, T. Mizusaki, and T. Otsuka, Phys. Rev. Lett. 77, 3315 (1996).

[5] F. Iachello and A. Arima, The Interacting Boson Model (Cambridge University Press, Cambridge, England, 1987).

[6] J. P. Elliott and A. P. White, Phys. Lett. 97B, 169 (1980).

[7] J. P. Elliott, J. A. Evans, and A. P. Williams, Nucl. Phys. A469, 51 (1987).

[8] M. J. Thompson, J. P. Elliott, and J. A. Evans, Nucl. Phys. A504, 436 (1989).

[9] M. Abdelaziz, J. P. Elliott, M. J. Thompson, and J. A. Evans, Nucl. Phys. A503, 452 (1989).
[10] J. A. Evans, G. L. Long, and J. P. Elliott, Nucl. Phys. A561, 201 (1993)

[11] V. S. Lac, J. P. Elliott, J. A. Evans, and G. L. Long, Nucl. Phys. A587, 101 (1995).

[12] J. N. Ginocchio and M. W. Kirson, Nucl. Phys. A350, 31 (1980).

[13] A. E. L. Dieperink and O. Scholten, Nucl. Phys. A346, 125 (1980).

[14] J. Dukelsky et al., Nucl. Phys. A425, 93 (1984).

[15] J. N. Ginocchio and A. Leviatan, Phys. Rev. Lett. 73, 1903 (1994).

[16] T. Otsuka, A. Arima, F. Iachello, and I. Talmi, Phys. Lett. 76B, 139 (1978)

[17] G. Colò, M. A. Nagarajan, P. Van Isacker, and A. Vitturi, Phys. Rev. C 52, R1175 (1995).

[18] P. J. Ennis et al., Nucl. Phys. A535, 392 (1991).

[19] J. N. Ginocchio, Phys. Rev. Lett. 77, 28 (1996).

[20] J. Engel, K. Langanke, and P. Vogel, Phys. Lett. B 389, 211 (1996). 